\title{
ESTILOS DE LIDERAZGO EN CARGOS UNIVERSITARIOS, ESTUDIO DESCRIPTIVO CON MUJERES DIRECTIVAS ${ }^{1}$
}

\author{
Leadership Styles in University Positions: Descriptive Study with Female Managers
}

Estilos de liderança em cargos universitários, estudo descritivo com mulheres diretivas

RECIBIDO:3 DE JULIO DE 2015

Bibiana Carolina Moncayo Orjuela

(Colombia)

Universidad Militar Nueva Granada

bibiana.moncayo@unimilitar.edu.co

\section{es}

\section{RESUMEN}

El acceso de mujeres a cargos de dirección en las instituciones de educación superior se convirtió en una importante línea de investigación entre los estudios de género y liderazgo. El propósito del artículo es describir la relación entre las variables sociales y demográficas con los estilos de liderazgo de mujeres a cargo de la dirección universitaria. Para esto se aplicó una encuesta a una muestra por conveniencia de 90 mujeres en cargos directivos en universidades acreditadas de Bogotá (Colombia). El estudio destaca que las mujeres con estudios doctorales y con estado civil divorciada/separada están asociadas a un estilo de liderazgo transformacional. La investigación pone de manifiesto la incidencia de factores sociales y demográficos que permiten estimular la participación femenina en el liderazgo educativo.
EVALUADO: 25 DE AGOSTO DE 2015

ACEPTADO:9 DE OCTUBRE DE 2015

\author{
David Zuluaga Goyeneche \\ (Colombia) \\ Universidad Militar Nueva Granada \\ david.zuluaga@unimilitar.edu.co
}

\section{en}

por

\section{ABSTRACT}

Women's access to leadership positions at higher education institutions has become an important subject of research within gender and leadership studies. The purpose of this article is to describe the relationship there is between demographic and social variables and the leadership styles of the women in charge of managing a university. In order to do this, we applied a survey to a convenient sample of 90 women in a managerial position at universities in Bogotá, Colombia. The study highlights that women with $\mathrm{PhDs}$ and who are divorced/separated are tied to a transformational leadership style. This investigation also displays the influence of social and demographic factors that encourage feminine participation in educational leadership.
PALABRAS CLAVE: mujeres, gestión educativa, liderazgo, variables sociales y demográficas.
KEYWORDS: Women, education management, social and demographic variables.

\section{RESUMO}

O acesso de mulheres a cargos de direção nas instituições de educação superior converteu-se em uma importante linha de pesquisa entre os estudos de género e liderança. $\mathrm{O}$ propósito do artigo é descrever a relação entre as variáveis sociais e demográficas com os estilos de liderança de mulheres a cargo da direção universitária. Para isto aplicou-se uma enquete a uma amostra por conveniência de 90 mulheres em cargos diretivos em universidades acreditadas de Bogotá (Colômbia). O estudo destaca que as mulheres com estudos doutorais e com estado civil divorciada/separada estão associadas a um estilo de liderança transformacional. A pesquisa põe de manifesto a incidência de fatores sociais e demográficos que permitem estimular a participação feminina na liderança educativa.

PALAVRAS CHAVE: : mulheres, gestão educativa, liderança, variáveis sociais e demográficas.

\section{Para CITAR ESTE ARTíCULO/TO CITE THIS ARTICLE/PARA CITAR ESTE ARTIGO:}

Moncayo Orjuela, B. C. y Zuluaga Goyeneche, D. (2015). Estilos de liderazgo en cargos universitarios, estudio descriptivo con mujeres directivas. Panorama, 9(17) pp. 74-84 . 


\section{INTRODUCCIÓN}

$\mathrm{Al}$ igual que en otros espacios, en las instituciones educativas, los hombres asumen (casi implícitamente) la acumulación del poder en vista de su trayectoria histórica y cultural de liderazgo. Aquello que Glazer-Raymo (1999) denomina old boy's club retrata un círculo donde los académicos designan de manera amistosa la cooptación de poder, lo cual desemboca en la eliminación sistemática (aunque no necesariamente deliberada) de las mujeres (García de Cortázar y García de León, 1997).

Al considerar la participación femenina en las instituciones de educación superior (IES), resulta relevante reconocerla como un fenómeno reciente y complejo. Allí, por una parte, la mujer se acoge bajo el estereotipo femenino que resalta sus virtudes pedagógicas familiares (Carrasco, 2004), pero, por otra parte, se le discrimina para ascender a cargos de gestión y administración (Sánchez, 2009; Glazer-Raymo, 1999; García de Cortázar y García de León, 1997; Unesco, 2002).

Este documento pretende reflexionar sobre la noción de género y liderazgo de la administración educativa, explorando figuraciones particulares de la división sexual del trabajo (Bourdieu, 2000). Se busca así describir la relación de variables sociales y demográficas con los estilos de liderazgo de mujeres en cargos directivos dentro de universidades acreditadas de Bogotá (Colombia). Para ello, se utiliza una metodología cuantitativa, que bajo el muestreo por conveniencia (Alamino y Castejón, 2006) encuesta a una población de noventa mujeres directivas en las IES seleccionadas.

Los análisis muestran que el perfil de directivas encuestadas en las IES tiende a describir una mujer con estilo de liderazgo transaccional: de 40 a 49 años, casada, con un promedio de 1.5 hijos y con estudio de maestría en Colombia. La mayoría accedió al cargo directivo principalmente por ascenso, promoción o concurso de méritos y dedican entre 9 y 11 horas diarias al desempeño de su labor directiva. Adicionalmente, gran parte de aquellas asociadas al liderazgo transformacional coinciden con la realización de estudio doctoral y estado civil solteral divorciada.

En conclusión, al vincular la temática del género y el liderazgo, se hace preeminente contemplar desde variables sociales y demográficas las condiciones profesionales y personales del cargo administrativo en la educación. Así es como se comprenden las particularidades de la problemática, y se obtendrán refuerzos para la superación de los continuos retos que enfrentan tanto las mujeres como los administradores en la educación superior.

\section{FUNDAMENTOS TEÓRICOS}

\section{LA IDENTIDAD Y EL GÉNERO}

A comienzos de la década de 1960, las ciencias sociales situaron la identidad como un proceso extenso y circunstancial que sienta sus bases en la socialización primaria del sujeto. Desde entonces la identidad sexual figura como una imagen del sujeto, asociada a la conciencia corporal colectiva. $\mathrm{Y}$ esta conduce a la interiorización de un sistema de símbolos que permiten al sujeto relacionarse con su entorno y los diferentes grupos de socialización. La masculinidad o feminidad no necesariamente devienen de la condición sexual, sino que conjuga una cantidad de factores socioculturales $\mathrm{y}$ contextuales alrededor de la pertenencia a un género (Bourdieu, 2000).

Inicialmente, y según Johnson (1965), la configuración de la identidad sexual tiene dos momentos clave: la identificación con el igual y la diferenciación con el otro. Es decir, la filiación de los hombres con su padre y la diferenciación con su madre, y viceversa para las mujeres. Un momento social que será transversal a los procesos sociales identitarios.

Posteriormente, surgen posturas antiesencialistas, que de manera alternativa rompen con la lógica dicotómica de la construcción social del género, y desvirtúan la validez del simple proceso de identificación y diferenciación. Se plantea que las divergencias entre hombres y mujeres no son objetivas, sino que, por el contrario, son creadas "performativamente" a través de procesos culturales, que se proclaman y se elaboran, teniendo la posibilidad de ser alteradas, desaparecidas, incluso declinadas.

Scott (1996) explica entonces que las relaciones sociales contienen un elemento basado en el género, el cual permea relaciones de poder y símbolos variantes a través del espacio-tiempo. Fraser (1995) complementa lo anterior

\section{Estilos de \\ liderazgo \\ en cargos \\ universitarios,}

estudio

descriptivo con mujeres directivas
I Panorama

| pp. $74-84$

I Volumen 9

I Número 17 I Julio-diciembre I 2015 
Bibiana Carolina

Moncayo

Orjuela I

David Zuluaga

Goyeneche I

Panorama I

pp. 74-84।

Volumen 9 I

Número 17 |

2015 |

\section{ESTILOS DE LIDERAZGO: EL LIDERAZGO EDUCATIVO}

Las teorías del liderazgo han pasado por tres momentos

al exponer que existe la posibilidad de deconstruir analíticamente la noción de género, en cuanto las identidades y las diferencias son construcciones discursivas ligadas a cada sujeto.

Judith Butler añade:

\begin{abstract}
Antes que una performance, el género sería performativo. [...] es, en efecto, una actuación, un hacer, y no un atributo con el que contarían los sujetos aún antes de su "estar actuando". [...] el género es una actuación reiterada y obligatoria en función de unas normas sociales que nos exceden. La actuación que podamos encarnar con respecto al género estará signada siempre por un sistema de recompensas y castigos. La performatividad del género no es un hecho aislado de su contexto social, es una práctica social, una reiteración continuada y constante en la que la normativa de género se negocia (Sabsay, 2009, p. 4).
\end{abstract}

Giddens (1989) explica también que los imaginarios de hombre/mujer son patrocinados por normas sociales que ejercen fuerte sugestión desde la crianza infantil y plantean presiones frente al cumplimiento de estereotipos. Particularmente en las mujeres, desde muy temprana edad, la socialización ha alentado competencias que favorecen la ejecución de roles maternales y maritales.

Bourdieu (2000) diría entonces que se ha naturalizado la existencia de un modelo legítimo androcéntrico, donde el orden social reafirma constantemente la dominación masculina. Y desde las instituciones se encuentra una "violencia simbólica" que con acciones discriminatorias, casi naturales, fomenta la escasa representación femenina en posiciones de poder.

La identidad sexual se entiende entonces como un pacto social, el cual opera en los sujetos de manera contundente. La persistencia y legitimidad del género es culturalmente constituida y constituyente y, mientras las instituciones reproduzcan estas dinámicas, la masculinidad y la feminidad seguirán siendo retroalimentadas por lógicas de poder inherentes (Bourdieu, 2000).

significativos, que incluyen las siguientes tendencias:
1. Personalidad del líder: su explicación tiene un fundamento mitológico o literario que recoge un enfoque heroico. Allí el líder es fundamentalmente una figura épica de trayectoria y contextura guerrerista, que se caracteriza por el logro de una gloria imperecedera antes que por el bienestar de sus subordinados (Carlyle, 1986).

2. Postura: vincula el liderazgo con sus seguidores. Se percibe entre ellos el imperativo del fenómeno relacional (Fiedler, 1967; Román y Ferri, 2013), y se establece que la autonomía de los seguidores para adherirse o no al líder radica en dos aspectos: el carisma y el estado de necesidad. Mediante su carácter interactivo, estos dos elementos definen la percepción del líder carismático: "La persona en quien, por virtud de cualidades personales insólitas, parece encarnar la promesa o esperanza de salvación" (Tucker, 1976, p. 109).

3. Contexto: basado en un marco que afecta el carisma del liderazgo (referido al lider en potencia y a sus posibles seguidores). De acuerdo con esto, los miembros que conforman la organización, las diferentes variables económicas, culturales, sociales, políticas, entre otras, acabarán por figurar en las funciones del liderazgo (Román y Ferri, 2008).

Más recientemente, a partir de Bass y Avolio (1994), las autoras Vega y Zavala (2004) formulan una herramienta metodológica cuantitativa mediante la cual se realiza una aproximación al estilo de liderazgo de los encuestados. Para esto se refieren tres estilos de liderazgo:

A. Liderazgo transformacional: es aquel que responsabiliza y exalta a los propios seguidores, explorando el conflicto y la crisis para conocerla y transformarla (Panebianco, 1990). Este tipo de liderazgo induce a los seguidores a trascender los intereses particulares a través del carisma percibido (Nye, 2010).

B. Liderazgo transaccional: se refiere a los intereses individuales de los seguidores en una relación de castigo-recompensa. El líder está en capacidad de crear incentivos comunes y establecer unas reglas para alcanzarlos (Vega y Zavala, 2004).

C. Liderazgo laissez-faire: es la ausencia de liderazgo que faculta a la organización a un curso espontáneo e imprevisto (Vega y Zavala, 2004).

Para finales del siglo XX, la organización educativa adopta constructos de liderazgo transformacional (Bass y Avolio, 1994), que perciben a los directivos educativos como agentes de cambio con capacidades transformadoras (Sánchez, 2009). De momento, esta postura ha llegado a ser denominada postransformacional, ya que retoma la tendencia "transformacional” e incluye la distribución de las capacidades alrededor de la organización (Riveros-Barrera, 2012). 
No obstante, otros autores sostienen que a la fecha el verbo liderar no ha sido claramente diferenciado del verbo gerenciar, ya que ambos se han entendido como un conocimiento, capacidad, habilidad o destreza adquirida para alcanzar un buen nivel de desempeño organizacional (Lorenzo, Sola y Cáceres, 2007). El escaso rigor en la fundamentación del liderazgo lo ha idealizado en calidad de figura imprescindible y fórmula recetaría.

El popular auge del liderazgo ha favorecido nuevas tendencias, en las que se concibe la solución a todos los problemas organizacionales (Bolman y Deal, 1995). Por ello, es importante tener precaución respecto de aquellas concepciones de significados nebulosos, que conllevan la conveniencia personal, el relativismo y el salvacionismo (Lorenzo, 2005).

Lorenzo (2005) propone entonces concebir el liderazgo educativo dentro de una visión poliédrica para designar estilos de liderazgo, quel logre abarcar variedad de contenidos en el universo semántico de la pedagogía. Su definición es esta: "La función de dinamización de un grupo o de una organización para generar su propio crecimiento en función de una misión o proyecto compartido" (p. 371).

\section{GÉNERO Y LIDERAZGO}

La temática sobre género y liderazgo se estudia con mayor énfasis en las últimas dos décadas, puntualizando respecto del sesgo masculino del liderazgo organizacional, aquello que Sánchez (2009) denomina gramática masculina del liderazgo. No obstante, dentro de las más recientes investigaciones, el concepto de género no ha logrado trascender versiones reduccionistas y diferencias dicotómicas hombre/mujer. En general, el género se asocia inevitablemente a lo femenino: una categoría no problemática y homogénea, donde todas las mujeres pueden ser enmarcadas dentro del estereotipo femenino.

Los resultados investigativos sobre género y liderazgo frecuentemente han quedado estancados en posiciones simplistas, en las que la función sexo define casi matemáticamente el estilo de liderazgo (Sánchez, 2009). Estas básicamente se orientan a determinar el comportamiento tanto del hombre como de la mujer en cargos directivos resaltando más en el sexo que en el género.
En esta misma línea, se ha perfilado un carácter ideológico carente de una base empírica sólida y de resultados concluyentes (Alimo-Metcalfe, 1995; Butterfield y Grinnell, 1999; Riehl y Lee, 1996; Eagly y Johnson, 1990), sesgados en la idealización del liderazgo femenino.

Según Blackmore (1996), se precisa abandonar la constante exploración respecto de las diferencias de género en el ejercicio de liderazgo, y encaminarse más bien a la manera en que las normas del género regulan el comportamiento organizacional. Reconociendo así que los distintos tipos de liderazgo constituyen "performatividades" masculinas y femeninas en culturas organizativas (Alvesson y Billing, 1992).

Resulta complejo afirmar que en las IES existe un liderazgo de hombres y otro de mujeres, asociado a estereotipos masculinos (competitividad, agresividad, determinación y autoritarismo) y femeninos (pasividad, emotividad y sensibilidad) (Andruskiw, 1980). Admitir que los hombres personifican un estilo de liderazgo masculino mientras que las mujeres asumen un tipo de liderazgo femenino es apresurado, puesto que cualquiera de los dos estilos puede ser, sino ejercido, adoptado por ambos sexos (Oplatka, 2006).

De cualquier forma, y evitando una posición radical, también es cierto que las tendencias del liderazgo femenino han sido efectivas y han logrado posicionarse entre las entidades de educación superior. Estas instituciones enfrentan un momento difícil de demandas externas, en las que las mujeres repetidamente ofrecen alternativas y propuestas organizacionales más democráticas, participativas y basadas en la ética del cuidado. Estas proposiciones han resultado, en ocasiones, mejores que los esquemas masculinos tradicionales en declive, asociados al autoritarismo, la ambición y la búsqueda del poder (Sánchez, 2009).

ACCESO FEMENINO A CARGOS DIRECTIVOS: CONTEXTO EDUCATIVO

Para el ámbito organizacional, llama la atención una mayoritaria presencia de mujeres en la base de la pirámide organizacional; sin embargo, el caso de las instituciones educativas demuestra una exclusión femenina de cargos directivos, que se ha mantenido bastante más 
Bibiana Carolina

Moncayo

Orjuela I

David Zuluaga

Goyeneche I

Diez, Terrón y Anguita (2009) usan la metáfora del "techo de cristal" para estudiar el posicionamiento directivo de las mujeres en instituciones educativas; figura que explica un entramado de obstáculos invisibles, mediante el que se hace alusión a la limitación de las mujeres calificadas para llegar a posiciones de poder dentro de las organizaciones (Roldán, 2012). El techo de cristal asemeja la aparente posibilidad de escalar laboralmente en la organización, coartada por barreras invisibles.

Hawley, Torres y Rasheed (1998) y Swanson y Witke (1997) caracterizan estas barreras en dos grupos:

1. Las barreras externas: des-estímulos directos o indirectos a causa del sexo, más comúnmente llamados discriminación.

2. Barreras internas (o culturales): se encuentran arraigadas a la esfera cultural, y otorgan poca tolerancia al cambio. Se distinguen como nociones de carácter idiosincrásico (casi mítico), que, antes de tener un contenido argumentativo, tienen un arraigado significado tradicionalista.

Las barreras externas son componentes sociodemográficos y contextuales que establecen las diferencias manifiestas del estereotipo masculino/femenino. Este tipo de barreras hacen parte de un imaginario colectivo de liderazgo, que, desde una dimensión simbólica, acaba definiendo la efectividad del género en la administración (Oplatka, 2006). Tomás y Guillamon (2009) exponen que a partir de allí se evidencian las normas sociales del juego que dejan en inferioridad de condiciones a las mujeres y las relegan al cuidado de la familia y el hogar.

Panorama I pp. 74-84 I

Volumen 9 I

Número 17 |

Estas barreras resultan más explícitas que las segundas en el sentido de que materializan un nivel de desigualdad en oportunidades de ascenso femenino. $Y$ aunque han intentado ser combatidas de manera oficial, infortunadamente no han logrado más que regular algunas condiciones estructurales.

Las barreras de tipo interno por su parte son decisivas en cuanto materializan una serie de papeles y preferen- identifican como comportamientos socialmente deseables en la mujer, afines con el sentido del deber, la voluntad de servicio y la falta de competitividad o ambición frente al poder.

En Cubillo y Brown (2003), las barreras internas experimentadas por las mujeres están fundamentadas en creencias culturales y religiosas. Allí el estereotipo femenino se acoge voluntariamente a un menor estatus y una remuneración inferior respecto de la de los hombres en las organizaciones. Díez, Terrón y Anguita (2009) de manera análoga denominan este fenómeno miedo al éxito; que en el contexto educativo explica una mayoritaria participación de mujeres en la profesión docente (Tomás y Guillamon, 2009).

Se reconoce así que la categorización de las barreras es efectiva para el entendimiento de la escasa representación de la mujer en posiciones de liderazgo, porque separa aquellos atributos autoimpuestos (Oplatka, 2006) de aquellos establecidos por el contexto (Acker, 1989; Coffey y Delamont, 2000; Limerick y Anderson, 1999).

\section{Metodología}

\section{TIPO DE INVESTIGACIÓN}

La metodología aplicada es de carácter descriptivo, y se basa en la recolección de datos cuantitativos. Se aplicó el muestreo por conveniencia (Alamino y Castejón, 2006), que por definición selecciona únicamente los casos disponibles. Es una estrategia no probabilística válida para la recolección de datos, en especial, con muestras pequeñas y muy específicas. A pesar de que los resultados de este tipo de prueba se limitan a generalizaciones entre la muestra estudiada, la potencia del muestreo está en seleccionar casos ricos en información para estudios en profundidad.

Este se escogió por dos motivos: 1) la población está compuesta por profesionales con poca disponibilidad de tiempo y atención a particulares y 2) algunas de las instituciones se acogen a la Ley Estatutaria 1581 de 2012 de Colombia, referida a disposiciones generales sobre protección de datos personales, que dificultan el acceso a los detalles de contacto. 


\section{MÉTODO DE INVESTIGACIÓN}

Se diseñó un instrumento tipo encuesta, cuya estructura se desagregó en seis partes:

1. Características demográficas

2. Perfil socioeconómico

3. Trayectoria académica

4. Situación laboral

5. Concepto del cargo actual

6. Estilo de liderazgo

Según Sánchez (2009) y Miranda y Ramírez (2011), se elaboró el instrumento metodológico, evaluado mediante una prueba piloto en siete universidades con doce mujeres directivas, y la revisión de pares académicos.

El instrumento final contó con veintidós preguntas, de las cuales dieciséis fueron de selección múltiple y cinco utilizaron la escala de Likert. Para complementar el muestreo por conveniencia, se aplicaron entrevistas a profundidad con tres de las directivas encuestadas.

\section{POBLACIÓN}

Se contó con la totalidad de mujeres directivas (en rectoría, vicerrectoría, decanatura, vicedecanatura, coordinación, dirección, secretaría y, en general, cargos de gobierno y toma de decisiones) de once IES acreditadas (Consejo Nacional de Acreditación, 2015) de Bogotá y Chía, correspondientes a una población de aproximadamente 283 académicas. La generación de la base de datos tuvo como insumo contactos tanto oficiales (suministrados por seis áreas de "talento humano") como no oficiales (extraídos de los directorios y páginas web de las dos instituciones restantes).

\section{TAMAÑO DE LA MUESTRA}

Una vez recopilados los datos de contacto de la población, se envió la encuesta mediante un correo en formulario "google.docs" a toda la población. El $31.8 \%$ (90 mujeres) de la población respondió la encuesta. Los datos de la muestra por conveniencia fueron procesados a través del programa estadístico SAS (tabla 1).

Tabla 1. Respuesta de instrumento según Universidad

\begin{tabular}{|c|l|l|l|}
\hline$N^{\circ}$ & Institución & $\begin{array}{l}\text { Valor } \\
\text { absoluto }\end{array}$ & $\begin{array}{l}\text { Valor } \\
\text { relativo } \\
\text { (ifras en } \\
\text { porcentaje }\end{array}$ \\
\hline 1 & $\begin{array}{l}\text { Universidad Nacional } \\
\text { de Colombia }\end{array}$ & 16 & 18 \\
\hline 2 & $\begin{array}{l}\text { Universidad de los } \\
\text { Andes }\end{array}$ & 5 & 5 \\
\hline 3 & $\begin{array}{l}\text { Pontificia Universidad } \\
\text { Javeriana }\end{array}$ & 27 & 30 \\
\hline 4 & $\begin{array}{l}\text { Universidad } \\
\text { Externado de } \\
\text { Colombia }\end{array}$ & 13 & 14 \\
\hline 5 & $\begin{array}{l}\text { Universidad del } \\
\text { Rosario }\end{array}$ & 19 & 21 \\
\hline 6 & $\begin{array}{l}\text { Dirección Nacional de } \\
\text { Escuelas }\end{array}$ & 4 & 4 \\
\hline 7 & $\begin{array}{l}\text { Universidad Santo } \\
\text { Tomás }\end{array}$ & 6 & 7 \\
\hline & Total & 90 & 100 \\
\hline
\end{tabular}

\section{RESULTADOS}

El perfil de mujeres directivas en las IES en general es de un rango de 40 a 49 años, estado civil casada, 1.5 hijos en promedio, vivienda propia ubicada en el estrato 41, disposición de empleada doméstica, estudio de maestría en Colombia, un rango de 1 a 3 años en el cargo directivo actual y de 7 a 10 años en la institución educativa, un rango de 1 a 3 años de experiencia en cargos directivos en las IES y un tiempo de experiencia total de 16 a 25 años.

La mayoría accedió al cargo directivo principalmente por ascenso, promoción o concurso de méritos, dedica entre 9

I pp. 74-84

I Volumen 9

I Número 17

I Julio-diciembre | 2015

1 En Colombia, los estratos son una clasificación socioeconómica de las viviendas o los predios que varia de 1 a 6 .
Estilos de

liderazgo

en cargos

universitarios,

estudio

descriptivo

con mujeres

directivas
Panorama (n) 
y 11 horas diarias al cargo y pertenece al área de la salud (28.89\%) o al área de ciencias humanas $(14.44 \%)^{2}$.

Bibiana Carolina

Moncayo

Orjuela I

David Zuluaga

Goyeneche I

Respecto del estilo de liderazgo, y según los postulados de Bass y Avolio (1994), se observa que el tipo de liderazgo preponderante dentro de la muestra por conveniencia fue el "estilo transaccional" $(55.55 \%)^{3}$. Se sugiere, entonces, que en efecto las directivas académicas encuestadas no están necesariamente adscritas a un tipo de liderazgo feminizado, donde resalta la pasividad, la emotividad y la sensibilidad (Alvesson y Billing, 1992). Por el contrario, el género femenino muestra diversos matices en los estilos de liderazgo, que están definidos por el contexto y las performatividades individuales.

En concordancia con lo que plantean autores como Alimo-Metcalfe (1995), Butterfield y Grinnell (1999), Riehl y Lee (1996) y Eagly y Johnson (1990), las mujeres no están inevitablemente asociadas a un estilo de liderazgo transformacional por su condición sexual. Los resultados reiteran los postulados de las teorías de género, donde "la performatividad de los individuos sexuados hace parte de su contexto social, es una práctica social, una reiteración continuada y constante en la que la normativa de género se negocia" (Sabsay, 2009).

Respecto de los resultados particulares de la encuesta, se presentan los datos más llamativos, a partir de cruces entre variables de interés. En primer lugar, y dentro de los detalles obtenidos a partir de la relación entre las variables sociales y demográficas con los estilos de liderazgo, se encuentra que el último nivel de estudio y el estilo de liderazgo están estrechamente asociados (figura 1).

Panorama I

pp. 74-84 I

Volumen 9 I

Número 17।

2 Otros estudios en España y Costa Rica concuerdan en que la enfermería, el trabajo social y las ciencias sociales son departamentos/programas con un nümero de directivas predominante (Sänchez, 2009; Twombly, 1998).

3 Es este estilo, predominan las relaciones con una visión parcelada y estrecha del mundo, que muestran incapacidad de internalizar visiones distintas de la propia, o peor, absoluta despreocupación respecto del otro.

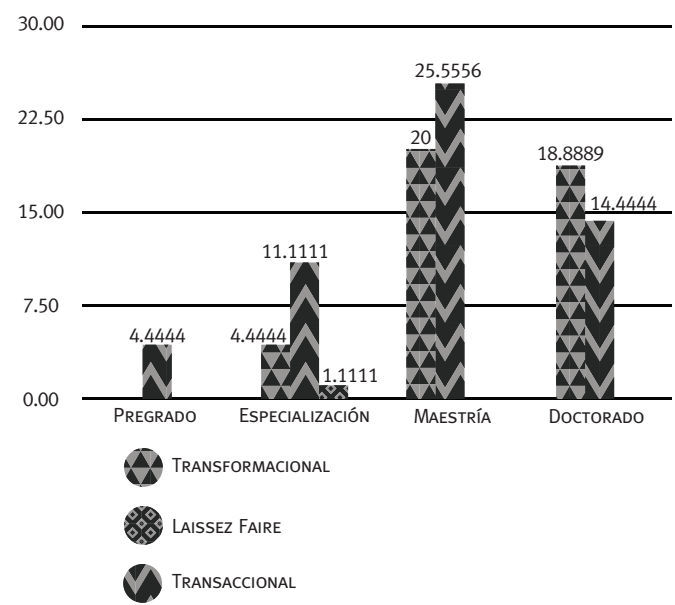

Figura 1. Estilo de liderazgo frente a último nivel de estudio.

Tal y como se aprecia en la figura 1, la mayor parte de las mujeres que pertenecen al estilo de liderazgo transaccional se asocian con estudio en pregrado (4.44\%), especialización (14.44 \%) y maestría (45.56\%). Sin embargo, aquellas con estudio de doctorado (33.33\%) presentan un mayor porcentaje para el estilo de liderazgo transformacional ${ }^{4}$.

Al contemplar correspondencia del nivel de estudio doctoral con el estilo de liderazgo transformacional, vale la pena considerar que en efecto existe "evidencia de una relación entre liderazgo transformacional y capital social" ${ }^{5}$ (Vega y Zavala, 2004, p. 10).

Considerando también el estrecho vínculo establecido entre el capital social y el capital cultural (referido grosso modo como nivel de estudio) (Bourdieu, 2000), cabe suponer que, gracias al estudio en posgrado (y las respectivas implicaciones socioculturales), las académicas encuestadas en efecto han alcanzado un estilo de liderazgo transformacional. Mediante su estudio superior ellas se han involucrado en medios de interacción ligados al bienestar público, que, en últimas, les han otorgado una dimensión de humanidad con su grupo de trabajadores, entre los cuales despiertan admiración e inspiración (Vega y Zavala, 2004).

En segundo lugar, resulta también interesante el cruce de las variables estado civil y último nivel de estudio (tabla 2).

\footnotetext{
4 Según Vega y Zavala (2004) este estilo se caracteriza por "un continuo mejoramiento en los repertorios conductuales y potencialidades tanto de los individuos como de los equipos. [...] pudiendo incluso llegar a desarrollar lideres internos. [...] Los seguidores (del lider transformacional) experimentan una transformación en sus creencias y valores, [...] para alcanzar el bien común, produciendo un aumento de su esfuerzo que supera las expectativas" (p. 11).

5 Concepto que se refiere a la cantidad de relaciones interpersonales que van conformando la comunidad civil, la estructura social y a partir de las cuales se constituyen redes efectivas para perseguir intereses (Cuoto, 1997).
} 


\begin{tabular}{|c|c|c|c|c|c|c|c|c|c|c|c|c|}
\hline \multirow{3}{*}{$\begin{array}{c}\text { Último nivel } \\
\text { educativo }\end{array}$} & \multicolumn{12}{|c|}{ Estado civil } \\
\hline & \multicolumn{2}{|c|}{ Casada } & \multicolumn{2}{|c|}{$\begin{array}{l}\text { Divorciada o } \\
\text { separada }\end{array}$} & \multicolumn{2}{|c|}{ Soltera } & \multicolumn{2}{|c|}{ Unión libre } & \multicolumn{2}{|c|}{ Viuda } & \multicolumn{2}{|c|}{ Total } \\
\hline & Total & $\%$ & Total & $\%$ & Total & $\%$ & Total & $\%$ & Total & $\%$ & Total & $\%$ \\
\hline Admón. policial & 1 & 1.1 & & & & & & & & & 1 & 1.11 \\
\hline Doctorado & 16 & 17.7 & 5 & 5.5 & 4 & 4.4 & 4 & 4.4 & 1 & 1.1 & 30 & 33.3 \\
\hline Especialización & 9 & 10 & 1 & 1.1 & 3 & 3.3 & & & & & 13 & 14.4 \\
\hline Maestría & 27 & 30 & 2 & 2.2 & 6 & 6.6 & 4 & 4.4 & 2 & 2.2 & 41 & 45.5 \\
\hline Pregrado & 1 & 1.1 & 1 & 1.1 & 1 & 1.1 & & & & & 3 & 3.3 \\
\hline $\begin{array}{l}\text { Subespecialidad } \\
\text { médica }\end{array}$ & & & & & 1 & 1.1 & & & & & 1 & 1.1 \\
\hline Subespecialización & 1 & 1.11 & & & & & & & & & 1 & 1.1 \\
\hline Total & 55 & 61.1 & 9 & 10 & 15 & 16.6 & 8 & 8.8 & 3 & 3.3 & 90 & 100 \\
\hline
\end{tabular}

Como se observa en la tabla 2 , de $61 \%$ de mujeres casadas, $30 \%$ de ellas cursó una maestría, $17.78 \%$ lo hizo en doctorado, $10 \%$ realizó estudios en especialización y solo $3 \%$ efectuó estudios en pregrado u otro nivel educativo. Entre $10 \%$ de mujeres separadas/divorciadas, $5.56 \%$ cursó un doctorado, $2.22 \%$ lo hizo en maestría y $2.22 \%$ lo llevó a cabo en especialización o pregrado.

Haciendo la salvedad respecto de la cronología de las variables aquí contempladas y las diferencias en proporción, para estos dos grupos mayoritarios (casadas y separadas/divorciadas), vale la pena destacar que, mientras entre las primeras poco menos de la mitad efectuaron estudios en maestría, entre las segundas, un poco más de la mitad cursó estudios en doctorado.

Se intuye entonces que, posiblemente una vez las mujeres casadas alcanzan la maestría, tienden a detener su estudio, mientras aquellas separadas/divorciadas tienden a continuar con estudio doctoral.

Según una de las directivas entrevistadas:

$$
\begin{aligned}
& \text { Para divorciarse usted tiene que romper con } \\
& \text { muchas cosas. Por ejemplo, tuve que romper } \\
& \text { con la Iglesia. Tuve que romper con la familia } \\
& \text { de mi papá. Mi papá, por su historia de vida, } \\
& \text { fue más probable que se encauzara por el } \\
& \text { lado de que no es verdad que la mujer tenga } \\
& \text { que someterse. (Si usted no estudia está } \\
& \text { condenada a depender siempre de alguien) } \\
& \text { (Entrevista personal, diciembre } 14 \text { de 2014). }
\end{aligned}
$$

\begin{tabular}{|c|c|c|c|c|c|c|c|c|c|c|c|c|}
\hline \multirow{3}{*}{ Personas a cargo } & \multicolumn{12}{|c|}{ Último nivel educativo } \\
\hline & \multicolumn{2}{|c|}{ Doctorado } & \multicolumn{2}{|c|}{ Especialización } & \multicolumn{2}{|c|}{ Maestría } & \multicolumn{2}{|c|}{ Pregrado } & \multicolumn{2}{|c|}{ Otros } & \multicolumn{2}{|c|}{ Total } \\
\hline & Total & $\%$ & Total & $\%$ & Total & $\%$ & Total & $\%$ & Total & $\%$ & Total & $\%$ \\
\hline $\begin{array}{l}\text { Hijos/as o } \\
\text { hijastros/as }\end{array}$ & 13 & 14.4 & 7 & 7.78 & 22 & 24.4 & 2 & 2.2 & 1 & 1.1 & 45 & 50.00 \\
\hline $\begin{array}{l}\text { Hijos o hijastros } \\
\text { y otros/as }\end{array}$ & 4 & 4.4 & 3 & 3.33 & 4 & 4.44 & 0 & & 2 & 2.2 & 13 & 14.44 \\
\hline $\begin{array}{l}\text { No tiene } \\
\text { personas a cargo }\end{array}$ & 11 & 12.2 & 2 & 2.22 & 13 & 14.4 & 0 & & 0 & & 26 & 28.89 \\
\hline Otros & 2 & 2.22 & 1 & 1.11 & 2 & 2.22 & 1 & 1.1 & 0 & & 6 & 6.67 \\
\hline Total & 30 & 33.3 & 13 & 14.44 & 41 & 45.5 & 3 & 3.3 & 3 & 3.3 & 90 & 100.0 \\
\hline
\end{tabular}

En tercer lugar, se advierte una relación interesante entre el último nivel de estudio y las personas a cargo de las encuestadas (tabla 3 ).

Tabla 3. Personas a cargo frente a último nivel de estudio 
Bibiana Carolina

Moncayo

Orjuela I

David Zuluaga

Goyeneche I

Según esto, para las mujeres los hijos/as podrían considerarse un obstáculo para continuar con otros estudios, y de ahí que la mayoría de ellas tienden a culminar programas de maestría. Por su parte, las mujeres sin hijos/ as a cargo tenderían a finalizar programas de doctorado con mayor facilidad. No obstante, es pertinente ser cautelosos con la información presentada, dado que no necesariamente son los hijos/as a cargo quienes representan un obstáculo para el estudio superior de las académicas. Probablemente, la situación refiere más bien la falta de apoyo en el cuidado diario de los hijos/as por parte de la pareja u otros miembros del hogar. Una situación que ilustraría la carga social y familiar que se le otorga a la madre.

Para las directivas encuestadas, se presume que es necesario culminar estudios de posgrado antes o después del periodo en que están a cargo de sus hijos. Por ello, como madres se encuentran en la disyuntiva entre hallar una edad adecuada donde no tengan una edad ni muy corta ni muy avanzada para continuar con sus cursos de nivel superior.

\section{CONSIDERACIONES FINALES}

En general, es necesario ser cuidadoso con las hipótesis anteriores debido a que son solo aproximaciones a un fenómeno social complejo. Los presupuestos aquí presentados son sujetos al debate y al rebate, y tienen como objetivo estimular la investigación en tan compleja temática.

No obstante, a partir de la información recogida, vale la pena destacar, como hallazgo principal, que no se detectó una relación directa entre las mujeres y el liderazgo transformacional, hecho que concuerda con algunos de los postulados de Alimo-Metcalfe (1995), Butterfield y Grinnel (1999), Riehl y Lee (1996) y Eagly y Johnson (1990) sobre la problemática. Por el contrario, se percibió que factores como el nivel de estudio de las académicas podría estar más asociado a su estilo de liderazgo.

Por esta razón, se hace necesario abordar la problemática desde una base contextual que permita leer el género como un elemento en interrelación con un sinnúmero de variables sociales. A partir de los elementos aquí abordados, se amplía el panorama de la problemática y los denominadores en común al respecto.

La problemática planteada amerita un análisis exhaustivo que evidencie y comprenda el diferencial de distribución de poder académico entre los respectivos géneros. El dilema ha estado opacado por una falsa ilusión de meritocracia organizacional que reina en las IES (Díez, Terrón y Anguita, 2009; Sánchez, 2009) que legitima y reproduce un contexto notoriamente feminizado pero tradicionalmente patriarcalista. Es necesario, entonces, evitar la reflexión que supone el asunto como una cuestión de tiempo, dado que hace varias décadas las mujeres conquistaron el medio académico y la situación hegemónica masculina continúa (García de Cortázar y García de León, 1997). Los cargos de gestión/administración en la educación superior no solo dirigen y coordinan la investigación y docencia, sino que también alcanzan una influencia política. Por ello, la selección y el reclutamiento de los directivos académicos están caracterizados por una elección de perfiles que trascienden el mérito para permear aspectos socioculturales que finalmente son influidos por la producción académica universitaria.

La hegemonía masculina sostiene unas dimensiones complejas con permanencias de largo alcance, que para el caso se manifiestan en rígidos procesos de inclusión a cargos de poder académico universitario. Tal y como se presenta en los resultados, más allá de evidenciarse una "discriminación manifiesta" contra las académicas, se perciben unas dinámicas de poder con cimientos que nos remiten a la estructura familiar. Se sugiere, a partir del presente ensayo, por tanto, revisar la participación femenina en la academia desde aspectos, como el estado civil y la maternidad. Estos elementos permiten que el análisis trascienda hacia consideraciones sociales con fundamentos enraizados en uno de los pilares de la sociedad moderna (la familia). De la mano, se invita a considerar la hipótesis del nivel de estudios en posgrado como un elemento movilizador de las académicas no solo respecto del mérito profesional, sino también 
en el sentido que puede alentar la puesta en marcha de habilidades asociadas al liderazgo transformacional y el bienestar organizacional.

Por todo lo anterior, se reconoce nuevamente la necesidad de una lectura interpretativa y exhaustiva de base contextual, que desde la propia academia transcienda y abarque procesos de exclusión e inclusión en la Universidad. Posiblemente es así que se develarán procesos de acceso al poder académico, con el fin de concientizar respecto de dicha problemática, tanto a las instituciones educativas como al género femenino.

\section{REFERENCIAS BIBLIOGRÁFICAS}

1. Acker, S. (1989). Teacher, Gender and Careers. Londres: The Falmer Press.

2. Alamino, A. y Castejón, J. (2006). Elaboración, análisis e interpretación de encuestas: cuestionarios y escalas de opinión. Recuperado de http:// rua.ua.es/dspace/bitstream/10045/20331/1/ Elaboraci \%C3 \%B3n \%2c \%20 an $\% \mathrm{C} 3 \%$ A1lisis \%20e \%20interpretaci $\%$ C3 \%B3n.pdf

3. Alimo-Metcalfe, B. (1995). An Investigation of Female and Male Constructs of Leadership and Empowerment. Women in Management, 10(2), 3-8.

4. Alvesson, N. y Billing, Y. (1992). Gender and Organization: Towards a Differentiated Understanding. Organization Studies, 13(12), 73-102.

5. Andruskiw, O.H. (1980). Dispelling a Myth: That Stereotypic Attitudes Influence Evaluations of Women as Administrators in Higher Education. The Journal of Higher Education, 51(5), 475-496.

6. Bass, B. y Avolio, B. (1994). Improving Organizational Effectiveness through Transformational Leadership. Thousand Oaks, CA: Sage Publications.

7. Blackmore, J. (1996). Breaking the Silence: Feminist Contributions to Educational Administration and Policy. En K. Leithwood, J. Chapman, D. Corson, P. Hallinger y A. Hart, International Handbook of Educational Leadership and Administration. Dordrecht: Kluwer Academic Publishers.

8. Bolman, C. y Deal, T.(1995). Organización y liderazgo. Wilmington: Adisson Wesley.

9. Bourdieu, P. (2000). La dominación masculina. Barcelona: Anagrama.
10. Butterfield, D. y Grinnell, J. (1999). "Reviewing" Gender, Leadership and Managerial Behavior: Do Three Decades of Research Tell us Anything? Handbook of Gender and Work. Thousands Oaks: Sage.

11. Carlyle, T. (1986). Los héroes: el culto de los héroes y lo heroico en la historia. México: Porrúa.

\section{Estilos de liderazgo en cargos}

12. Carrasco, M.J. (2004). Análisis de los estilos directivos de las mujeres en centros educativos. Revista Iberoamericana de Educación, 33(3), 1-13.

13. Coffey, A. y Delamont, S. (2000). Feminism and the Classroom Teacher: Research, Praxis, Pedagogy. Londres: Routledge/Falmer.

14. Consejo Nacional de Acreditación (2015). Consultar instituciones acreditadas. Recuperado de http://www.cna.gov.co/1741/article-186354. html

15. Correa, M. (2005). La feminización de la educación superior y las implicaciones en el mercado laboral y los centros de decisión politica. Recuperado de http://mineducacion.gov.co/cvn/1665/articles-92159_archivo_pdf.pdf

16. Cubillo, L.y Brown, M. (2003). Women into Educational Leadership and Management: International Differences? Journal of Educational Administration, 41(3), 278-291.

17. Cuoto, R. (1997). Social Capital and Leadership. En S. Webster (ed.), Transformational Leadership Working Papers: Kellogg Leadership Studies Project. College Park, MD: The Burns Academy of Leadership, University of Maryland.

18. Díez, E., Terrón, E. y Anguita, R. (2009). Percepción de las mujeres sobre el "techo de cristal” en educación. Revista Interuniversitaria de Formación de Profesorado, 23(1), 27-40.

19. Eagly, A. y Johnson, B. (1990). Social Role of the Theory of Sex Differences and Similarities: A Current Appraisal. Psychological Bulletin, 108(2), 233-256.

20. El, U. y Jonnergård, K. (2010). Included or Excluded? The Dual Influences of the Organisational Field and Organisational Practices on New Female Academics. Gender and Education, 22(2), 209-225.

21. Fiedler, F. (1967). A Theory of Leadership Effectiveness. Nueva York: McGraw-Hill.

I Panorama

I pp. $74-84$

22. Fraser, N. (1995). Multiculturalidad y equidad de los géneros: un nuevo exámen de los debates en torno a la diferencia en EE. UU. Recuperado de http://www.mujeresenred.net/IMG/pdf/ Multiculturalidad_y_equidad_entre_los_ generos.pdf
I Volumen 9

I Número 17 I Julio-diciembre | 2015 
23. García de Cortázar, M. y García de León, M. (1997). Mujeres en minoría: una investigación sociológica sobre las catedráticas de Universidad en España. Madrid: Centro de Investigaciones Sociológicas.

Bibiana Carolina

Moncayo

Orjuela I

David Zuluaga

Goyeneche 1

Glazer-Raymo, J. (1999). Shattering the Myths: Women in Academe. Baltimore: The Johns Hopkins University Press.

26. Hawley, E., Torres, D. y Rasheed, S. (1998). Assessing Barriers to Women's Career Adjustment. Journal of Career Assessment, 6(4) 449-479.

27. Johnson, H. (1965). Sociología, una introducción sistemática. Buenos Aires: Paidós.

28. Leithwood, K. (1994). El liderazgo para la reestructuración de las escuelas. Revista de Educación, 304, 31-60.

29. Limerick, B. y Anderson, C. (1999). Female Administrators and School-Based Management. Educational Management and Administration, 27(4), 401-414.

30. Lorenzo, M. (2005). El liderazgo en las organizaciones educativas. Revista Española de Pedagogía, 232, 367-388.

31. Lorenzo, M., Sola, T. y Cáceres, M. (2007). E1 liderazgo femenino en los cargos directivos: un estudio longitudinal en la Universidad de Granada (1990-2005). Educación y Educadores, 10(2), 177-194.

32. Miranda, N. y Ramírez, L. (2011). ¿Quiénes dirigen las instituciones educativas en Cali?: perfil de los directivos docentes en colegios públicos y privados de estratos 1 a 4 . Educación y Educadores, 14(3), 559-576.

33. Noer, D. (1997). El cambio en las organizaciones. México: Prentice May.

34. Nye, J. (2010). Leadership e Potere: Hard, Soft, Smart Power. Bari: Laterza.

35. Oplatka, I. (2006). Women in Educational Administration within Developing Countries: Towards a New International Research Agenda. Journal of Educational Administration, 44(6), 604-624.

2015 |

36. Panebianco, A. (1990). Modelos de partido. Madrid: Alianza.
37. Riehl, C.y Lee, V. (1996). Gender Organizations and Leadership. International Handbook of Educational Leadership and Administration. Dordrecht: Kluwer Academic Publishers.

38. Riveros-Barrera, A. (2012). La distribución del liderazgo como estrategia de mejoramiento institucional. Educación y Educadores, 15(2), 289-301.

39. Roldán, E. (2012). Segregación laboral y techo de cristal. Recuperado de http://www.academia.edu/6490614/ Segregacion_laboral_y_techo_de_cristal_en_ trabajo_social_analisis_del_caso_espanol

40. Román, P. y Ferri, J. (2008). La participación política y el empoderamiento social de las mujeres. En: P. Román (coord.), Pobreza, mujeres y medio ambiente. Madrid: Fundación IPADE-UCM.

41. Sabsay, L. (2009). Judith Butler para principiantes. Recuperado de http://www.pagina12.com.ar/ diario/suplementos/soy/1-742-2009-05-09.html

42. Sánchez, M. (2009). mujeres dirigentes en la Universidad: las texturas del liderazgo. Zaragoza: Colección Sagardiana.

43. Scott, J. (1996). El género, una categoría útil para el análisis histórico. En M. Lamas, El género: la construcción cultural de la división sexual (pp. 265-302). México: PUEG.

44. Swanson, J. y Witke, M. (1997). Theory into Practice in Career Assessment for Women: Assessment and Interventions Regarding Perceived Barriers. Journal of Career Assessment, $5,443-462$.

45. Tomás, M. y Guillamon, C. (2009). Las barreras y los obstáculos en el acceso de las profesoras universitarias a los cargos de gestión académica. Revista de Educación, 350, 253- 275.

46. Tucker, R. (1976). La teoría del liderismo carismático. En D. A. Rustow (ed.), Filósofos y estadistas. México: FCE.

47. Twombly, S. (1998). Women Academic Leaders in a Latin American University: Reconciling the Paradoxes of Professional Lives. Higher Education, 35(4), 367-397.

48. Unesco (2002). Women and Management in Higher Education: A Good Practice Handbook. París: Unesco.

49. Vega, C.y Zavala, G. (2004). Adaptación del cuestionario multifactorial de liderazgo (MLQ forma 5x corta) de B. Bass y B. Avolioal contexto organizacional chileno (Tesis de grado, Universidad de Chile). Recuperado de http:// www.tesis.uchile.c1/tesis/uchile/2004/vega_c/ sources/vega_c.pdf 